PREPARED FOR SUBMission to JHEP

\title{
Non-spherical collapse in AdS and Early Thermalization in RHIC
}

\author{
Eunseok $\mathrm{Oh}^{a}$ and Sang-Jin $\mathrm{Sin}^{a}$ \\ ${ }^{a}$ Department of Physics, Hanyang Univ. Seoul 133-791 \\ E-mail: lspk.lpg@gmail.com, sjsin@hanyang.ac.kr
}

\begin{abstract}
In the flat space, non-spherical shells collapse to give globular cluster after many oscillations. We show that in anti de sitter space, they form black holes in one dynamical time. We propose that this is the mechanism of early thermalization in strong quark-gluon plasma in gravity dual. This is traced back to the a remarkable property of AdS : the period in radial motion is amplitude independent in spite of the NON-linearity of the equation of motion. We investigate the interaction effect numerically and observe the same qualitative behavior for the attractive forces. For repulsive interactions, particles halt at a small but finite radius for long time due to the specific structure of the bulk AdS propagator. It helps hair creation in the AdS black hole.
\end{abstract}




\section{Contents}

1 Introduction 1

2 Collapse in global $\mathbf{A d S}_{5}$ without inter-particle interaction 2

3 Flat boundary case $\quad 5$

3.1 Effect of the initial velocity 5

4 The effect of the Interaction $\quad 6$

$\begin{array}{lll}5 & \text { Conclusion } & 7\end{array}$

\section{Introduction}

One of the mysteries of RHIC experiment is the early thermalization[1]. The fireball made from the collision seems to reach equilibrium in $1 \mathrm{fm} / \mathrm{c}$, which is comparable time for gold ions to pass each other. This is attributed to the strongly interacting nature of the system. Since it is hard to understand this phenomena in perturbative field theory, it is natural to ask if a dual formulation where particles are weakly coupled is helpful. According to the gauge/gravity duality [2-4], thermalization is dual to the black hole formation in Anti de Sitter(AdS) space. In a previous paper of one of the authors [5], mapping the entire process of RHIC experiment to the dual gravity language was tried. Our question in this formulation is "Why is the black hole formation time of a generic gravitational collapse in AdS space of the order of the passing time of the two ions?" In other words, "Why does generic gravitational collapse produce black hole in one falling time in AdS space?"

For the collapse of a spherical shell, it is less surprising that the final result is the black hole. However, for non-spherical shell, the result is far from a black hole in flat space, where a globular cluster rather than a black hole is formed. Recent studies on scalar field collapse in AdS space [6-10] shows that even for the spherical symmetric collapse, black hole is made only after repeated reflections from the boundary. Therefore such results have some distance from 'thermalization in one passing time'. The natural explanation for the experiment should be such that the black hole is formed in one falling time for any generic initial configuration.

Also, showing the black hole formation by numerical analysis does not give an 'understanding' why it is formed so easily in AdS but not in flat space. While some analytic discussion is provided in [7], it is not very clear to us what the physical mechanism of inevitable black hole formation is. Confining or Box nature of AdS space does NOT explain the easy formation of black hole.

In this paper, we will study the collapse of non-spherical shell made of dusts, and show that it forms a black hole in one finite falling time due to a specific property of the AdS. 
We treat the shell as a collection of dust particles, which is very different from the scalar field collapse where it is assumed that matter is in a state of coherent condensation. Only when particle's wave functions are overlapping, one can justify treating many particles in terms of condensation wave function or the scalar field configuration.

In the gauge/gravity duality one considers the limit where string is much smaller than the AdS radius. In this limit the wave nature as well as the stringy nature is suppressed. For the many particle system in RHIC, a scalar field configuration is not the proper dual configuration to the fireball. Therefore we consider the shell as a collection of the particles in the global AdS. We will show that particles arrive at the center simultaneously regardless of their masses and initial positions as far as they start from static configurations. This means that any non-spherical shell in AdS space becomes more spherical shell as it falls, and all parts of the shell reach the center simultaneously. We call this property as synchronization effect of AdS. After such shell pass the 'would be horizon', black hole forms and particle's motion can not and need not be traced if we take into account the back reaction of the gravity. It proves that any cloud falls to become a black hole in AdS space.

After this we consider the phenomena in the Poincare patch where AdS/CFT is manifest. We will see that there will be a finite time which is less than one falling time at which all the particle are inside the radius of apparent horizon as far as there is no IR cut off in the geometry. We will first show this for particles without inter-particle interaction; then we will show that the same thing is true even in the presence of the interaction. In gauge/gravity duality, the dynamics of gluon exchange is treated by gravity background in leading order of $1 / \mathrm{N}$ expansion. So here neglecting inter-particle interaction means treating particle interaction only in leading $1 / \mathrm{N}$ expansion of gauge theory.

Even in the simplest case of free fall along the radial direction, the equation of the motion (EOM) is that of a non-linear oscillator. Nevertheless its period is independent of the amplitude as if it is a simple harmonic oscillator(SHO). This is a remarkable property of AdS spacetime. We will de-mystify this phenomena by finding a non-linear mapping that transforms the EOM of the falling into that of SHO. In fact, the idea of Synchronization was first formulated in ref. [5] where it was observed that the radial motion is that of SHO in the proper time and in Poincare coordinate. However each particle has its own proper time and therefore it could not be claimed definitely that particles arrive at the center at the same time based on the "same falling proper time". Also in that paper a geometry with IR brane describing the confining phase was used, which can raise the question: how a system in confining phase can go to a deconfining phase by cooling? As it was argued in [5], the falling is dual to the expansion which cause the system to cool down. One of the purpose of this paper is to improve these difficulties.

\section{Collapse in global $\mathrm{AdS}_{5}$ without inter-particle interaction}

To consider the collapse of non-spherical shell which consists of non-interacting particles, we need to look at the motion of individual particles. For $A d S_{5}$ with spherical boundary 
the metric is given by

$$
d s^{2}=-\left(1+r^{2} / R^{2}\right) c^{2} d t^{2}+r^{2} d \Omega^{2}+\frac{d r^{2}}{1+r^{2} / R^{2}}
$$

It is well known that the falling time from the boundary to the center following the null geodesic is $\pi R / 2 c$. However we will see that even the massive particle starting from arbitrary position with zero radial velocity will arrive at the center after the same time. Notice that the time for the light starting from the arbitrary position to arrive at the center will not be the same.

The equation of motion is given by the action

$$
S=-m \int \sqrt{-g_{\mu \nu} \dot{x^{\mu}} \dot{x^{\nu}}} d t \text {, with } \dot{x}=\frac{d x}{d t} .
$$

For simplicity, we first consider the radial motion and set $R=1, c=1$. The energy conservation can be written as

$$
\frac{m\left(1+r^{2}\right)}{\sqrt{1+r^{2}-\dot{r}^{2} /\left(1+r^{2}\right)}}=E .
$$

It is easy to see that the system describes a non-linear oscillator. Notice that the dot in the above equation is derivative in $t$ not in the proper time unlike ref. [5]. If we assume that the particle starts with zero radial velocity from the initial radial position $r_{0}$, then

$$
E=m \sqrt{1+r_{0}^{2}}
$$

which establishes a dictionary between the total energy and the initial radial coordinate. Introducing $v_{c}$ by $v_{c}=r_{0} / \sqrt{1+r_{0}^{2}}$, we have

$$
E=\frac{m}{\sqrt{1-v_{c}^{2}}}
$$

Its velocity in the radial direction starts with 0 and become $v_{c}$ when it arrive at the center. Interestingly, we can find the exact solution of the equation of motion:

$$
r=\frac{v_{c} \cos t}{\sqrt{1-v_{c}^{2} \cos ^{2} t}}
$$

The remarkable property of this solution is that the period of the motion is $2 \pi$, independent of the original position $r_{0}$, as if it is a simple harmonic oscillator. Restoring the scale parameters $R, c$ by $r, t, v \rightarrow r / R, t / R, v / c$ the falling time is

$$
T_{\text {fall }}=\frac{\pi}{2} \frac{R}{c}
$$

This means that arbitrary set of particles, falling in AdS will form a black hole regardless of the initial position, provided, (i) all the particles start with zero initial velocities, and (ii) Interaction between the particles are negligible. See figure 1.

How do we justify or relax the conditions listed above? 

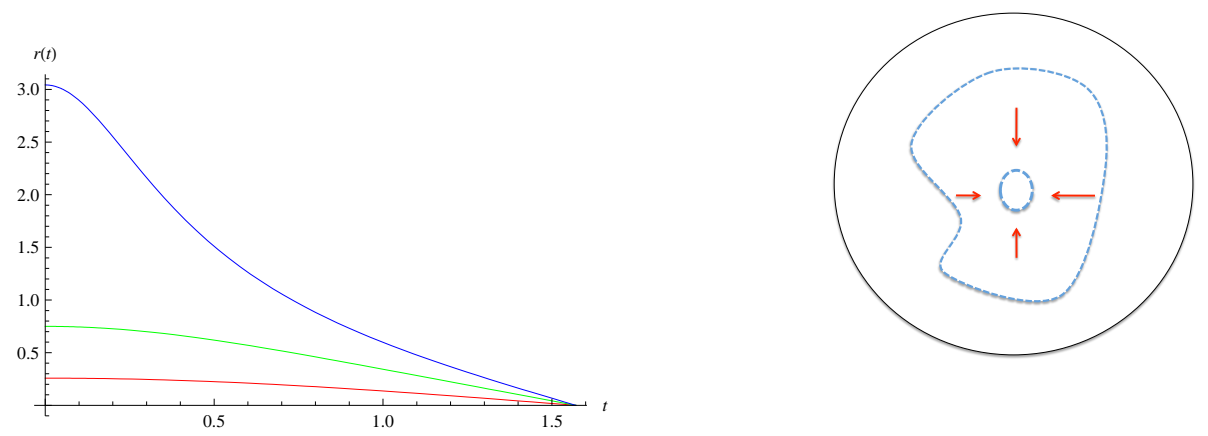

Figure 1. Left: Free falling of a particle in AdS for various initial positions. Falling time is independent of the initial position; Right: Collapse of a shell with arbitrary shape in AdS space. The whole shell will reach at the center simultaneously. $T_{\text {fall }}=\frac{\pi}{2} \frac{R}{c}$.

1. In AdS/CFT correspondence, the radial direction is the dual of the energy scale. If two gold ions collided in RHIC and created particles of energy $E_{i}$ and mass $m_{i}$, $i=1, \cdots, N$ inside the fireball, then the holographic image of such fireball is the particles in AdS space at the position

$$
r_{0 i}=\sqrt{\left(E_{i} / m_{i}\right)^{2}-1} .
$$

Particles created in the fireball have velocities in $x^{i}$ directions but not in radial direction. Therefore their dual image particles do not either. This justifies the first issue listed above.

2. In gauge/gravity dual, the gluon dynamics is replaced by the gravitational background and the leading order inter-particle gluonic interaction in the many body quark-gluon plasma is approximated as the particle motions in a fixed gravitational background. Interparticle interaction in AdS is necessary only to take care of the non-gluon interaction or non-leading order interaction in $\mathcal{O}\left(1 / N_{c}\right)$; therefore, such inter particle interaction in AdS bulk should be absent or very weak in the large $N_{c}$ theory.

To de-mystify the amplitude independence of the period of the above nonlinear oscillators, we can actually map the above non-linear oscillator equation to that of the harmonic oscillator. If we define $\eta$ by

$$
r=\frac{\eta}{\sqrt{1-\eta^{2}}}
$$

the eq.(2.3) can be mapped to the harmonic oscillator;

$$
\dot{\eta}^{2}+\eta^{2}=\eta_{0}^{2}:=1-\left(\frac{m}{E}\right)^{2},
$$


whose solution is $\eta=\eta_{0} \cos t$. In this coordinate, the metric becomes

$$
d s^{2}=\frac{1}{1-\eta^{2}}\left(-d t^{2}+\eta^{2} d \Omega^{2}\right)+\left(\frac{d \eta}{1-\eta^{2}}\right)^{2} .
$$

We can also define $\eta=\tanh \rho$ to get $r=\sinh \rho$ to get the metric in the global coordinate:

$$
d s^{2}=-\cosh ^{2} \rho d t^{2}+d \rho^{2}+\sinh ^{2} \rho d \Omega^{2} .
$$

The point is that once the particles pass the would-be horizon of the system, there is no turning back. Apparent horizon formation is inevitable consequence of the synchronized falling.

\section{Flat boundary case}

Now we work in Poincare patch where gauge/duality is well established. The metric is given by

$$
d s^{2}=\frac{r^{2}}{R^{2}}\left(-c^{2} d t^{2}+d \mathbf{x}^{2}\right)+R^{2} \frac{d r^{2}}{r^{2}}
$$

and it has flat boundary. The integrated equation of motion is

$$
\frac{m r^{2}}{\sqrt{r^{2}-\dot{r}^{2} / r^{2}}}=E
$$

and its solution is [5]

$$
r=\frac{\epsilon}{\sqrt{1+(\epsilon t)^{2}}}
$$

This is not a periodic solution. It show that starting from $r_{0}=E / m=\epsilon$, it takes infinite time to reach the center. However, the large time behavior, $r \sim 1 / t$, is independent of the initial height $r_{0}=\epsilon$. This is a manifestation of the synchronization effect in this metric: two particles with different initial height fall and get closer, which is enough to argue the canonical formation of the black hole in this metric. This effect was observed in [5] as a 'tendency' of time focusing. However, due to the infinite falling time in Poincare coordinate time, it was not recognized there that synchronization is the exact property of the AdS space, partly because IR cut off was introduced in the work. ${ }^{1}$ Later, we will show that introducing the interaction does not change the synchronization property of AdS.

\subsection{Effect of the initial velocity}

Here we study the effect of the initial velocity along the space time direction. Suppose the particle is in motion along $x$ direction. Then two first integrals are

$$
\frac{m r^{2}}{\sqrt{r^{2}\left(1-\dot{x}^{2}\right)-\dot{r}^{2} / r^{2}}}=E, \frac{m r^{2} \dot{x}}{\sqrt{r^{2}\left(1-\dot{x}^{2}\right)-\dot{r}^{2} / r^{2}}}=p,
$$

\footnotetext{
${ }^{1}$ The concept of synchronization was initiated in [5] due to the fact that the radial equation of motion in the Poincare patch was found to be simple harmonic oscillator in the proper time. However, each particle has its own proper time and it could not be concluded that exact synchrozation is the property of AdS from this either. Notice that the SHO discovered in this paper is in real time not proper time.
} 
which can be called as the energy and momentum respectively. If we set $V=p / E$,

$$
r=\frac{\epsilon\left(1-V^{2}\right)}{\sqrt{1+\left(\epsilon\left(1-V^{2}\right) t\right)^{2}}} .
$$

we get $\dot{x}=V$. Remarkably the large time behavior of the radial position is independent of all of the initial conditions $m, E, p$. Therefore we can say that the time focusing effect is perfect even in the presence of the motion along the collisional direction.

\section{The effect of the Interaction}

So far, we discussed the particles falling without interaction. We now discuss the effect of it in Poincare coordinate where the metric is

$$
d s^{2}=\frac{1}{x_{0}^{2}}\left(d x_{0}^{2}+d x^{\mu} d x_{\mu}\right), \text { with } x_{0}=1 / r
$$

The scalar propagator for particle with mass $m^{2}=\Delta(\Delta-d)$ in the $\operatorname{AdS}_{d+1}$ is given in [12] and it is given by

$$
G \sim\left(\frac{1}{u(2+u)}\right)^{\Delta} \text { with } u=\frac{(x-y)^{M}(x-y)_{M}}{2 x_{0} y_{0}}
$$

Since $u$ is nonnegative and we are interested in the most singular contribution, we will neglect the factor $u+2$. The Newtonian potential in AdS can be derived from this to give

$$
\begin{aligned}
V\left(\left\{x_{i}, y_{i}\right\}\right) & =\iint d^{5} x d^{5} y J(x) G(x, y) J(y), \\
& =G_{N} \sum_{i<j} \int d t \frac{\left(x_{i 0} x_{j 0}\right)^{2 \Delta}}{\left(\left|x_{i}(t)-x_{j}(t)\right|^{2}+\left|x_{i 0}(t)-x_{j 0}(t)\right|^{2}\right)^{\Delta-1 / 2}}
\end{aligned}
$$

where $J(x)=\sum_{i} \delta^{4}\left(x^{A}-x_{i}^{A}(t)\right), A=0,1,2,3$. Since the initial velocities are only along the $x^{\mu}$ direction, we expect that the attractive interaction will only enhance the focusing effect in radial motion. Indeed we can verify this numerically, using the equation of motion derived from the Lagrangian

$$
S=-m \int \sqrt{-g_{\mu \nu} \dot{x^{\mu}} \dot{x^{\nu}}} d t,+V\left(\left\{x_{i}, y_{i}\right\}\right)
$$

In the numerical calculation we used $G_{N}=1, \Delta=3 / 2$ for simplicity.

In figure 2, falling of a few particles with some horizontal initial velocities starting from different heights are drawn. The calculation is done by the mathematica. The vertical lines are the falling trajectories and the horizontal dashed lines indicate the equal time slices. As time goes on, it is manifest that the radial positions converge. In figure 3 , we consider what happens if inter-particle interactions are repulsive. This is possible if particles carry extra charges. Interestingly, we see that falling is halted for some moments at the certain radial region and then proceeds to the black hole formation. We can understand such behavior from the structure of the propagator (4.2) : In the deep IR region, $u \rightarrow 0$ for any 


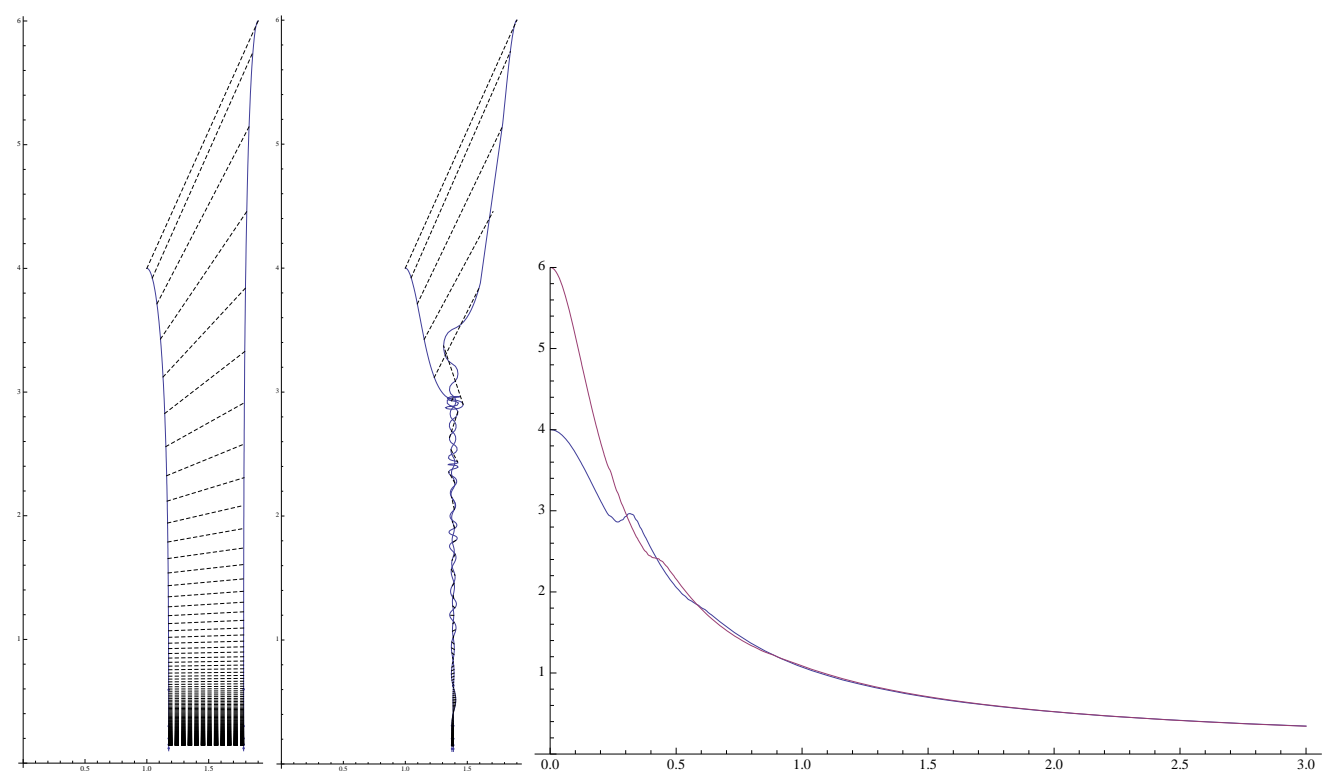

Figure 2. Falling in AdS with and initial velocity. : (Left) without inter-particle interaction. This is the flat-boundary analogue of Figure 1. (Middle) with interaction. $r$ v.s x, x-axis is one of the space direction. (Right) radius as a funcntion of time. The synchronization effect (equalizing the radial position) is manifest here.

finitely distant two points, therefore strong repulsion is effective there although particles are separated enough. We speculate that this can be the mechanism of the formation of the gravitational hair which is observed in the theory of holographic superconductor.

In figure 4, we show what happens if three particles collide with inter-particle attraction and repulsion. As we can see, whatever is the situation, radial positions of particles converges. Therefore we conclude that such synchronization effect is not destroyed by the interaction effect, especially if the interaction is attractive. For the repulsive case, particles halts at small but finite radius for long time due to the specific structure of the propagator: namely, $x_{0} y_{0}$ factor in $1 / u$. See (4.2) and (4.4). We believe that this is responsible for the presence of the hair in the AdS space.

\section{Conclusion}

In this paper we demonstrated that arbitrary shape of shell in AdS falls and form a black hole. The physical mechanism is the synchronized falling which is the characteristic property of AdS. While individual particle's motion is oscillation, many particle's motion should be terminated by the black hole formation. Once the black hole is formed, there will be no more oscillation. The details of the stabilization of the system through losing the potential energy should be worked out by considering the back reaction of the metric, which is not the scope of this work.

The reason for several oscillation before black hole formation in the study of the spherical scalar field collapse is due to the wave nature of the scalar field: as the field configuration collapses, uncertainty principle activates the kinetic term [11], which generates pressure and 


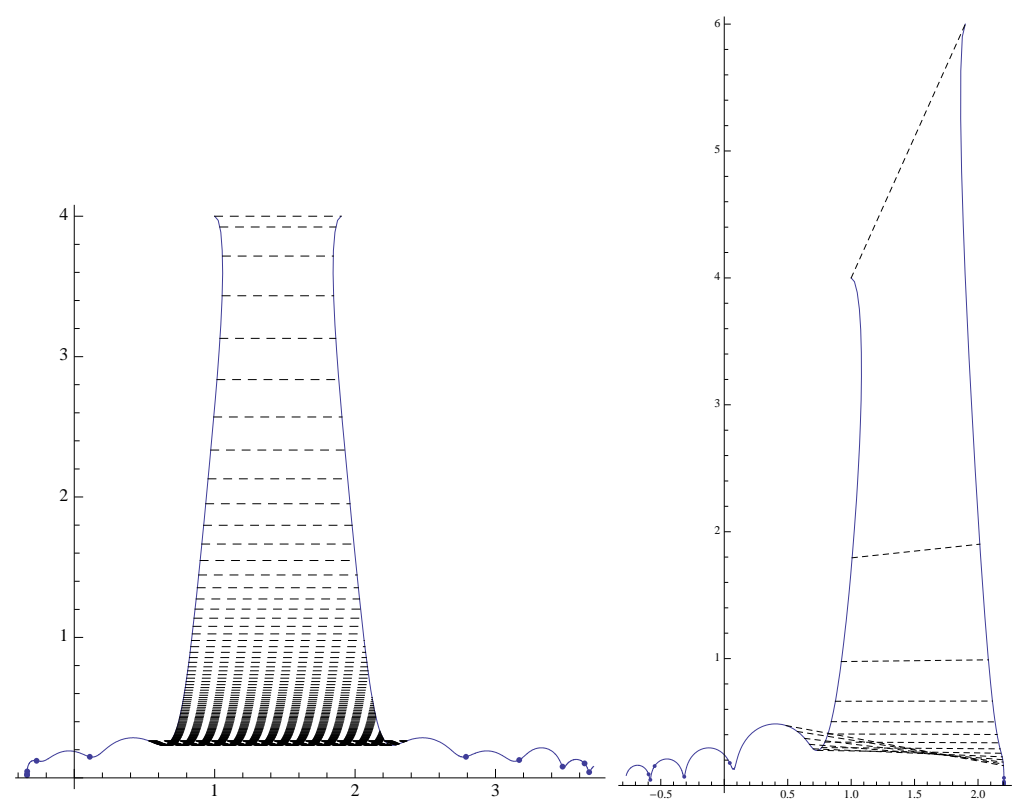

Figure 3. $r$ v.s $x$. Falling in AdS with repulsive interaction. L) start from the same height. R) start from the different height.

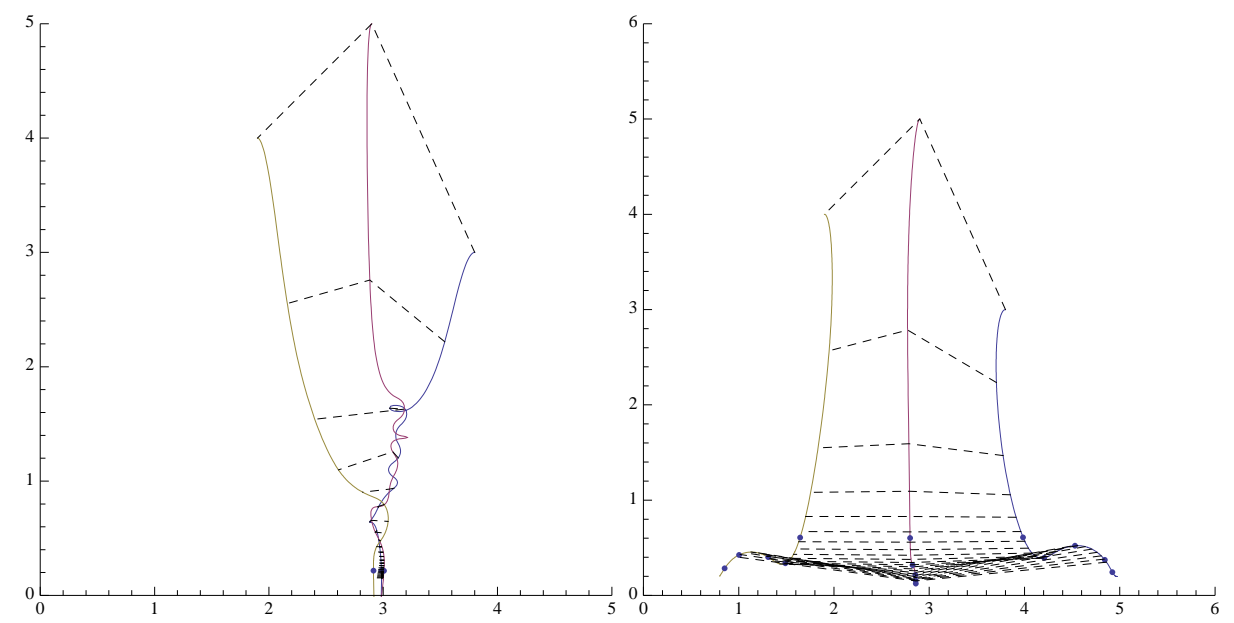

Figure 4. Three particles colliding in 5 dimension. L) with attractive interaction. R) with repulsion. All the particle arrive at the center simultaneously even in the presence of the interaction.

causes the bounce. It can be also attributed to the effective repulsion between the scalar particles as we have seen in the section 4 .

The future work is to find the effect of the extra interactions and to find the time dependent solution of Einstein equation that describes the formation of the black hole. Large time approximate solution was proposed in [14] and further developed in [15, 16]. However, finding exact Einstein gravity solution is non-trivial even for the spherically symmetric shell and we will postpone it to future work. 


\section{Acknowledgments}

This work was supported by Mid-career Researcher Program through NRF grant No. 201300000001340. It is also partly supported by the NRF grant through the SRC program CQUeST with grant number 2005-0049409.

\section{References}

[1] U. W. Heinz and P. F. Kolb, Nucl. Phys. A 702, 269 (2002) [hep-ph/0111075].

[2] J. M. Maldacena, The Large $N$ limit of superconformal field theories and supergravity, Adv. Theor.Math.Phys. 2 (1998) 231-252, [hep-th/9711200].

[3] S. Gubser, I. R. Klebanov, and A. M. Polyakov, Gauge theory correlators from noncritical string theory, Phys.Lett. B428 (1998) 105-114, [hep-th/9802109].

[4] E. Witten, Anti-de Sitter space and holography, Adv.Theor.Math.Phys. 2 (1998) 253-291, [hep-th/9802150].

[5] E. Shuryak, S. -J. Sin and I. Zahed, J. Korean Phys. Soc. 50, 384 (2007) [hep-th/0511199].

[6] S. Bhattacharyya and S. Minwalla, JHEP 0909, 034 (2009) [arXiv:0904.0464 [hep-th]].

[7] P. Bizon and A. Rostworowski, Phys. Rev. Lett. 107, 031102 (2011) [arXiv:1104.3702 [gr-qc]].

[8] D. Garfinkle and L. A. Pando Zayas, Phys. Rev. D 84, 066006 (2011) [arXiv:1106.2339 [hep-th]].

[9] D. Garfinkle, L. A. Pando Zayas and D. Reichmann, JHEP 1202, 119 (2012) [arXiv:1110.5823 [hep-th]].

[10] A. Buchel, L. Lehner and S. L. Liebling, Phys. Rev. D 86, 123011 (2012) [arXiv:1210.0890 [gr-qc]].

[11] S. -J. Sin, Phys. Rev. D 50, 3650 (1994) [hep-ph/9205208].

[12] E. D'Hoker and D. Z. Freedman, Nucl. Phys. B 550, 261 (1999) [hep-th/9811257].

[13] E. D'Hoker, D. Z. Freedman, S. D. Mathur, A. Matusis and L. Rastelli, Nucl. Phys. B 562, 330 (1999) [hep-th/9902042].

[14] R. A. Janik and R. B. Peschanski, Phys. Rev. D 73, 045013 (2006) [hep-th/0512162].

[15] S. Nakamura and S. -J. Sin, JHEP 0609, 020 (2006) [hep-th/0607123].

[16] S. -J. Sin, S. Nakamura and S. P. Kim, JHEP 0612, 075 (2006) [hep-th/0610113]. 